\title{
List of Reviewers 2010
}

Eastern Economic Journal (2011) 37, 305. doi:10.1057/eej.2011.1

The Editors and Editorial Board wish to acknowledge the assistance of the following colleagues, who reviewed articles during 2010, for their contributions of time and experience. Without their dedication, our work in publishing this Journal could not go forward.

Aamer Abu-Qarn

Richard Adelstein

Dori Allard

Peter von Allmen

Gene Amromin

Mikhail Anufriev

Pedro de Araujo

Nathan J. Ashby

Adam Atherly

Richard C. Barnett

Anders Bjorklund

Stacey L. Brook

Christopher Bruce

Thomas Bruggink

Philippe Burger

Jim Butkiewicz

Miles B. Cahill

Marco Capasso

Gaetano Carmeci

Sergio Cesaratto

Menzie David Chinn

Sofronis Clerides

Riccardo Corradini

Metin Cosgel

Pascal G. Courty

Lyn Craig

Bill Craighead

Dean Croushore

Vince Daly

Dhaval Dave

Lewis Davis

Craig Depken

James M. DeVault

Paul J. Devereux

Arcangelo Dimico

Oguzhan C. Dincer

Giovanni Dosi

Benoit Dostie

Stephen Drinkwater

Julan Du

Alex Dupuy

Dan Elfenbein

John Ermisch

Sarah M. Estelle

Arturo Estrella

T. Windsor Fields

Peter L. Francia

\begin{tabular}{|c|c|}
\hline Edoardo Gaffeo & Aaron Lowen \\
\hline Jordi Gali & Michael Douglas \\
\hline Thomas Garrett & Makowsky \\
\hline Domenico Delli Gatti & Svitlana Maksymenko \\
\hline Ross Gayler & Catherine Mann \\
\hline Anne Christina Gielen & Robert Andrew Margo \\
\hline Rita Ginja & Peter Hans Matthews \\
\hline Darren Grant & KimMarie McGoldrick \\
\hline Adam J. Grossberg & Ross McKitrick \\
\hline Lester Hadsell & Robin McKnight \\
\hline Rik Hafer & Monika Merz \\
\hline David R. Hakes & Richard A. Miller \\
\hline Wen-Jui Han & Ted R. Miller \\
\hline Nobuyuki Hanaki & Javier Miranda \\
\hline Kristiana M. Hansen & Madhu Sudan \\
\hline Alexander Haupt & Mohanty \\
\hline Mark G. Herander & Gary Mongiovi \\
\hline Duncan Hodge & Marie Mora \\
\hline Emily Hoffman & Swati Mukerjee \\
\hline Christiaan Hogendorn & Sean Eric Mulholland \\
\hline Ed Hopkins & Marina Murat \\
\hline Kai-Lung Hui & Peter Neary \\
\hline Jennifer Hunt & Kevin Stefan Nell \\
\hline Andreas Irmen & Todd Nesbit \\
\hline Wen-Jhan Jane & Romain de Nijs \\
\hline Susan M. Jellissen & Felix Oberholzer-Gee \\
\hline Byeongju Jeong & Alexandre Olbrecht \\
\hline Stefan Jungblut & Ann L. Owen \\
\hline Anneli Kaasa & Michael T. Owyang \\
\hline Pavel Kapinos & Amanda Pallais \\
\hline William D. King & Katherine Pancak \\
\hline Moris M. Kleiner & Susana Peralta \\
\hline Kim Klyver & Hans Pitlik \\
\hline James Konow & Florian Ploeckl \\
\hline Kajal Lahiri & Anne Polivka \\
\hline John C. Leadley & Andrew Potter \\
\hline Rick LeBlanc & Joshua Price \\
\hline Evelyn L. Lehrer & William Rand \\
\hline Wenli Li & Bhaskara Rao \\
\hline Ivan Light & Daniel A. Rascher \\
\hline Jason M. Lindo & Yaron Raviv \\
\hline Jody Lipford & Daniel I. Rees \\
\hline Gao Liu & Elke Renner \\
\hline Hakan Locking & Marta Reynal-Querol \\
\hline Dunia Lopez-Pintado & Kurt W. Rotthoff \\
\hline Ricardo Lopez-Ruiz & Isabel Ruiz \\
\hline David A. Love & David B. Ryden \\
\hline
\end{tabular}

Marc Santugini

Edward Scahill

Barry Scholnick

Jeremy T. Schwartz

Amit Sen

Mark Setterfield

Mark H. Showalter

Edward Shumacher

Peter Skott

Bill Smar

Russell S. Sobel

Stephen J. Spurr

Dean B. Stansel

Steven Stern

Thomas Stratmann

Yuki Takahashi

Simge Tarhan

Jeremy Thornton

Paul Trescott

Panos Tsakloglou

James A. Turner

Leanne J. Ussher

Arturo Ramirez

Verdugo

Stefania Villa

Nicolas Vincent

Andreas Waldkirch

Stephen J.K. Walters

David Waltz

Junbo Wang

Yongsheng Wang

Elizabeth Watson

Andrea Weber

Marshall White

Anne Winkler

Mark E. Wohar

Kip Womack

Susan Woodward

Stephen Wu

Andrew T. Young

Alejandro Zentner

Yan Zhao

Benjamin Chris

Zissimos 